\title{
1 Chancen der Stakeholder- und Marken- kommunikation im Rahmen eines ganzheitlichen Marketing-Managements nutzen
}

Hans-Peter Pohl

- Pro-aktive, Dialog-orientierte Kommunikation ist Kernaufgabe der Führung eines Forschungsverbundes in der globalisierten, vernetzten Welt

- Generisches Marketing-Verständnis als Führungsansatz

- Gelebte Identität und Kultur prägen Image und Reputation

- Stakeholder-Management und Markenpflege sind strategische Bausteine der Kommunikation

- Identifikation der relevanten Stakeholder in 5 Schritten (Makro-Analyse)

- Mikro-Analyse der relevanten Stakeholder

- Eine starke Marke vermittelt dem Forschungsverbund Sinn

- Ziel der Kommunikation: mit den Stakeholdern in den Dialog kommen

\subsection{Pro-aktive, Dialog-orientierte Kommunikation ist Kernaufgabe der Führung eines Forschungsverbundes in der globalisierten, vernetzten Welt}

Die Herausforderungen an die Forschungsverbünde in der globalisierten Welt sind vielfältig: Der weltweite, europäische und nationale Wettbewerb um öffentliche und private Forschungsförderung sowie um etablierte Wissenschaftler/innen und qualifizierte Nachwuchs-Wissenschaftler/innen nimmt zu. Der Effizienzdruck steigt. Hierarchien werden flacher, neue Formen der Zusammenarbeit z.B. in Netzwerken etablieren sich. Die Auf- 
gabenstellungen werden komplexer, Erkenntnisse und Informationen nehmen sprunghaft zu. Die Anspruchsgruppen (Stakeholder) zeigen sich immer kritischer, fordern Transparenz, mischen sich immer stärker ein. Neue Kommunikationsformen durch Internet und Soziale Medien sind Fluch und Segen zugleich. Diese und andere Entwicklungen stellen besondere Anforderungen an die effektive Führung einer Forschungseinrichtung bzw. eines Forschungsverbundes: Sie bedarf eines emphatischen, pro-aktiven, transparenten, Dialog-orientierten Informations- und daraus resultierenden Kommunikationsverständnisses. Es erfordert zudem die Fähigkeit, verbal und nonverbal den Verstand und die Gefühle der Stakeholder anzusprechen.

\subsection{Generisches Marketing-Verständnis als Führungsansatz}

Dieses moderne Kommunikations-Verständnis ist eingebettet in ein generisches Marketing- bzw. Marketing-Management-Verständnis als Führungsansatz einer Organisation, $z u$ dem es in den vergangenen Jahrzehnten von einer reinen Vertriebsfunktion entwickelt worden ist. Kotler (2011) hat diesen Wandel international entscheidend geprägt. National stehen Raffée, Fritz, Wiedmann (1994) für diese Entwicklung im Hinblick auf öffentliche Betriebe oder Fritz (1996) auf Hochschulen: „Marketing ist ein Prozess im Wirtschaftsund Sozialgefüge, durch den Einzelpersonen und Gruppen ihre Bedürfnisse und Wünsche befriedigen, indem sie Produkte und andere Dinge von Wert erzeugen, anbieten und miteinander austauschen." (Kotler et al. 2011, S. 39) „Marketing bezieht sich somit auf die Gestaltung von Austauschbeziehungen. Marketing-Management meint dagegen die Gesamtheit der Führungsaktivitäten, die auf die Gestaltung der Austauschprozesse abzielen." (Raffée et al. 1994, S. 35/36).

Fritz (1996) bezieht dabei wie Kotler und Raffée auch nicht nur die Austauschbeziehungen des Absatz-Markts (z.B. Wettbewerber, Unternehmen) ein, sondern integriert zusätzlich umfassend alle weiteren Austauschbeziehungen einer Hochschule des internen Markts (z.B. Professoren, Wissenschaftliche Mitarbeiter, Mitarbeiter aus Verwaltung und Technik, Studierende), des Beschaffungs-Markts (z.B. Schüler, Hochschul-Absolventen, Wissenschaftler, Arbeitnehmer, Banken, Aufsichts- und Genehmigungsbehörden, Banken, Dienstleister, Förderer, Kooperationspartner) und des Public-Markts (z.B. Politik, Verbände, Medien, Gesellschaftliche Gruppen).

\subsection{Gelebte Identität und Kultur prägen Image und Reputation}

Eine klare Positionierung und ein gelebtes Leitbild geben dem Forschungsverbund bei den internen Anspruchsgruppen (Management, Mitarbeiter, Beiräte, Gesellschafter, Träger, Mitglieder) Orientierung, Motivation und Anleitung zum Handeln, prägen seine Identität und auf Dauer seine Kul- 
1 Chancen der Stakeholder- und Markenkommunikation im Rahmen eines ganzheitlichen Marketing-Managements nutzen

tur. Im Idealfall nehmen die externen Anspruchsgruppen den Forschungsverbund auch entsprechend wahr. Deutlich wird dabei aber auch: Je diffuser Positionierung und Leitbild die Identität und Kultur des Forschungsverbundes prägen und damit nach außen erscheinen lassen, desto vielschichtiger und widersprüchlicher sind seine Wahrnehmung und Wertschätzung bei den externen Anspruchsgruppen (Image und Reputation).

\subsection{Stakeholder-Management und Markenpflege sind strategische Bausteine der Kommunikation}

Ein Forschungsverbund wird mit den Erwartungshaltungen unterschiedlichster Stakeholder konfrontiert: Interne wie Entscheidungsträger, Mitglieder, Manager und Mitarbeiter, externe wie Förderer, Spender, Sponsoren und Kooperationspartner, Medien und gesellschaftliche Interessensgruppen sowie staatliche Einrichtungen. Aufgabe des Stakeholder-Managements ist es, durch ständige Beobachtung und Interaktion die für den Forschungsverbund relevanten Stakeholder zu identifizieren und den Dialog zu suchen und zu pflegen, um deren Wahrnehmung im Sinne des Forschungsverbundes zu prägen. Dabei hilft eine konsistente Markenwahrnehmung als Referenzrahmen. Während Positionierung und Leitbild die Angebotsseite des Forschungsverbundes beschreiben, stellt die Marke das Vorstellungsbild dar, das sich in der Psyche der Stakeholder herausbilden soll: Mit welchen Botschaften wollen wir welche Vorstellungen bei den Anspruchsgruppen auslösen?

Mit den Bausteinen der Identität des Forschungsverbundes - Erscheinungsbild, Kommunikation und Verhalten der internen Stakeholder - prägt er das Vorstellungsbild in den Köpfen der externen Stakeholder. Der Nutzen einer starken Marke ist für die internen wie externen Stakeholder groß: Für den Forschungsverbund stellt sie ein Güte-, Qualitäts- und Vertrauenssiegel dar, differenziert ihn vom Wettbewerb, bildet Präferenzen bei den externen Anspruchsgruppen, stiftet bei ihnen und den internen Anspruchsgruppen Identifikation und Loyalität. Bei den externen Stakeholdern erleichtert eine starke Forschungsverbunds-Marke die Auswahl unter den Wettbewerbern, wird als Qualitätsversprechen und -Sicherung gewertet, trägt neben diesem Grundnutzen zusätzlich zu einem „psychologischen Zusatznutzen“ bei: zur Identifikations-, Image- und Statusbildung, wodurch Loyalität und Bindung mit dem Forschungsverbund sowie die Bereitschaft zu dessen Weiterempfehlung gefördert werden. 


\subsection{Identifikation der relevanten Stakeholder in 5 Schritten (Makro-Analyse)}

Aufgrund begrenzter Ressourcen wird sich ein Forschungsverbund auf die Kommunikation mit den relevanten Stakeholdern konzentrieren müssen (Freemann et al. 1983, S. 83ff.). Eine schrittweise Integration unterschiedlicher wissenschaftlicher Ansätze, die der Autor bei seiner Beratungs-Arbeit verfolgt hat, führt dabei zu einem effektiven Ergebnis:

In einem ersten Schritt gilt es unvoreingenommen alle möglichen internen und externen Stakeholder (Einzelpersonen, Gruppen, Organisationen) zu sammeln, die im direkten oder indirekten Bezug zum Forschungsverbund stehen bzw. stehen könnten.

Je breiter und tiefer die Zusammensetzung der Gruppen und die Repräsentanten der Organisationen durchdrungen werden, desto deutlicher kann deren Vernetzung untereinander in einem zweiten Schritt analysiert werden. So wird deutlich, dass die selben Einzelpersonen oder Gruppen häufig in unterschiedlichen Rollen zum Forschungsverbund in Beziehung stehen können.

In einem dritten Schritt werden in Anlehnung an Janisch (1992, S. 123) diejenigen Stakeholder als „Strategische Anspruchsgruppen“ herausgefiltert, denen Einfluss auf den Forschungsverbund zugesprochen werden kann, erst recht, wenn ihr Einfluss hoch ist und sie davon auch Gebrauch machen.

Im vierten Schritt sind diese „Strategischen Anspruchsgruppen“ in Anlehnung an Savage et al. (1991, S. 65) hinsichtlich ihrer Haltung zum Forschungsverbund zu analysieren: Sind sie eher unterstützend, neutral oder kritisch bis ablehnend?

Im fünften Schritt gilt es in Anlehnung an Müller-Stewens und Kollegen (2001, S. 270) zu klären, welche der Strategischen Anspruchsgruppen beeinflussbar sind und welche nicht.

Im Ergebnis kristallisieren sich diejenigen Strategischen Anspruchsgruppen als relevant heraus, die über großen Einfluss verfügen, diesen auch einsetzen und dem Forschungsverbund unterstützend zur Seite oder konstruktiv-kritisch gegenüberstehen. Nicht beeinflussbare, gegenüber dem Forschungsverbund ablehnend agierende strategische Anspruchsgruppen wie z.B. Wettbewerber oder Interessengruppen des Public-Markts sind zu beobachten. Ggf. sind vorsorglich Kommunikationsstrategien zur Eindämmung ihres „Bedrohungspotenzials“ zu entwickeln.

Diese Stakeholder-Analyse ist eine permanente Aufgabe, denn Kommunikationsbeziehungen sind fließend und unterliegen einer ständigen Veränderung. So können aktuell relevante Stakeholder an Bedeutung verlieren, zunächst weniger relevante an Bedeutung gewinnen. 
1 Chancen der Stakeholder- und Markenkommunikation im Rahmen eines ganzheitlichen Marketing-Managements nutzen

\subsection{Mikro-Analyse der relevanten Stakeholder}

Die relevanten Stakeholder, insbesondere Einzelpersonen und Entscheider in Organisationen, sind soweit wie möglich zu personalisieren und gegebenenfalls sozio-demografisch einzuordnen. In jedem Fall sollten aber ihre Einstellungen zum und ihre Erwartungshaltungen an den Forschungsverbund analysiert werden. Dialog-orientierte Kommunikation bemüht sich zunächst um dieses Verstehen der relevanten Stakeholder, ehe der Dialog gesucht wird bzw. er z.B. nach den Erwartungshaltungen fragend gestartet wird. Hierfür bieten sich berufliche Netzwerke wie XING oder LinkedIn, aber durchaus auch private Netzwerke wie Facebook oder Google+ an. Nach Veröffentlichungen und Meinungsäußerungen der Personen z.B. im Internet oder auch Facebook ist zu recherchieren. Kollegen oder Bekannte, die diese Personen näher kennen, können wertvolle Informationen geben.

\subsection{Eine starke Marke vermittelt dem Forschungsverbund Sinn}

Wie oben beschrieben, erleichtert eine starke Marke aufgrund ihrer positiven Wahrnehmung seitens der Stakeholder die Kommunikationsaufnahme mit ihnen. Neurowissenschaftliche Erkenntnisse belegen (Scheier u. Held 2007), dass nur 5\% aller Handlungsentscheidungen bewusst reflektierend erfolgen, dagegen 95\% quasi automatisch im Hintergrund geprägt werden. Marken haben diesbezüglich für den Forschungsverbund eine Doppelfunktion: Sie verschaffen ihm einerseits eine vom Wettbewerb differenzierende Bedeutung und motivieren andererseits zur Zusammenarbeit oder Nutzung (Belohnungsfunktion).

Grundlagen der Markenentwicklung sind die Positionierung und das Leitbild des Forschungsverbundes. Liegen sie nicht vor oder sind sie weiterzuentwickeln, ist dazu eine SWOT-Analyse zu erarbeiten: Die externen Bedingungen (Opportunities \& Threats) und die internen Faktoren (Strengths \& Weaknesses), denen die Entwicklung des Forschungsverbundes unterliegt, sind zu analysieren. Langfristige Trends, politische, kulturelle, gesellschaftliche und rechtliche Rahmenbedingungen, die Einstellungen und Erwartungshaltungen der externen Stakeholder, die Stärken und Schwächen der Wettbewerber verdeutlichen die externen Bedingungen des Marktes, in dem der Forschungsverbund tätig ist.

Die identifizierten Stärken und Schwächen des Forschungsverbundes sind diesen externen Bedingungen gegenüber zu stellen, sodass daraus abgeleitet werden kann, mit welchen Stärken die Chancen des Marktes genutzt werden können bzw. welche Ressourcen benötigt werden, um eventuelle Schwächen zu überwinden, die die Chancen-Nutzung behindern. Mögliche Risiken sind $\mathrm{zu}$ beachten, möglichst zu vermeiden oder realistisch einzuschätzen. Im Er- 
gebnis sind die strategische Positionierung und das Leitbild herauszuarbeiten:

- Was ist unsere Aufgabe?

- Was ist unser Angebot und was wollen wir gemeinsam erreichen?

- In welchem strategischen Geschäftsfeld und welchem (räumlichen) Marktgebiet sind wir aktiv?

- Mit welchen Kompetenzen, herausragenden Fähigkeiten und Ressourcen sind wir im Wettbewerb erfolgreich bzw. wollen wir erfolgreich sein?

- Welchen Nutzen stiften wir bzw. wollen wir stiften?

- Was zeichnet uns besonders aus bzw. soll uns besonders auszeichnen?

- Was ist Sinn unseres Handelns?

- Welche Prinzipien prägen unser Handeln?

- Was macht uns unverwechselbar, ggf. einzigartig?

Dürfte den meisten Forschungseinrichtungen oder Forschungsverbünden die Erarbeitung einer strategischen Positionierung und eines Leitbilds vertraut sein, stellt die Entwicklung der Marke eine häufig noch ungewohnte Aufgabe dar. Die Marketingwissenschaft hat dafür den identitätsorientierten Markenansatz entwickelt, der sich an der Wechselwirkung von Identität und Image orientiert (Burmann et al. 2012). Dabei wird einem Produkt oder Angebot bzw. einer Organisation wie einem Unternehmen, einer Forschungseinrichtung oder einem Forschungsverbund eine Persönlichkeit zugesprochen wie einer Person. Zu entwickeln ist die Identität dieser Markenpersönlichkeit. Sie ist dann so effektiv zu kommunizieren, dass sich in den Köpfen der Stakeholder ein mit der Identität kongruentes Image als Vorstellung herausbildet. So wie die Kommunikation der Marke die Persönlichkeit eines Menschen als Ganzes anspricht (Covey 1989), also seinen Körper, seinen Verstand, seine Emotion und zur Sinn-Stiftung sein philosophisches Zentrum (Kotler et al. 2010), so beinhaltet die Marke selbst auch diese Bausteine, die sich in Anlehnung an Gad (2005) in vier Dimensionen beschreiben lassen:

- Nutzen der Marke für den Nutzer (funktional)

- Identifikationsmöglichkeiten des Nutzers mit der Marke (mental)

- Möglichkeiten des Nutzers, mit der Marke in Dialog zu treten und/ oder die Marke als Teil seiner Persönlichkeit zum Ausdruck zu bringen (sozial)

- Besondere Verantwortung der Marke, die sie gegenüber Natur, Umwelt und Gesellschaft zum Ausdruck bringt (ethisch).

Mit Hilfe dieser vier Dimensionen wird das angestrebte Vorstellungsbild bei den Stakeholdern geprägt. Während die mentale und die ethische Dimension der Marke ihre Bedeutung geben, sie also vom Wettbewerb differenzieren, verdeutlichen die funktionale und die soziale Dimension den Belohnungscharakter der Marke. 
1 Chancen der Stakeholder- und Markenkommunikation im Rahmen eines ganzheitlichen Marketing-Managements nutzen

In einer vertiefenden Fokussierung sollte nach Gad die DNS der Marke herausgearbeitet werden:

- Angebotsvorteil: Welche Vorteile bietet unsere Marke den Nutzern?

- Positionierung: Warum ist unsere Marke anders oder besser als die der Konkurrenz?

- Stil: Was charakterisiert den Stil, das Image oder die Tonalität unserer Marke?

- Mission: Welche Rolle spielt die Marke in der Gesellschaft, oder welchen Vorteil hat die Marke für die Öffentlichkeit?

- Vision: Die Marke kann ihre eigene Zukunft definieren: Wo soll sie in x Jahren mit welchen Stärken stehen?

- Werte: Was macht unsere Marke so vertrauenswürdig wie einen Freund?

Aus den Antworten kann die Einzigartigkeit, die Unverwechselbarkeit der Marke als ihre Hauptidee, als ihren Markenkern, destilliert werden.

Angesichts der nachhaltigen strategischen Bedeutung der Marke und der Notwendigkeit, dass sie von den internen Stakeholdern eines Forschungsverbundes auch in ihrem täglichen Verhalten gelebt wird, sollten sie in die Markenentwicklung einbezogen werden.

\subsection{Ziel der Kommunikation: mit den Stakeholdern in den Dialog kommen}

Die operative Kommunikationsaufgabe unterscheidet sich von Stakeholder zu Stakeholder:

- Gilt es zunächst, die Marke überhaupt einem Stakeholder bekannt zu machen, ihn auf die Marke aufmerksam zu machen?

- Oder kennt er sie bereits, kann sie aber noch nicht richtig einschätzen, braucht daher profilbildende Informationen?

- Oder sollen mit Hilfe der Kommunikation konkrete Handlungen des Stakeholders ausgelöst werden?

- Oder soll er mit Hilfe der Kommunikation nach einem Informationsaustausch oder aufgrund einer Zusammenarbeit an den Forschungsverbund gebunden werden?

Jede dieser unterschiedlichen Aufgaben erfordert ein adäquates Kommunikationskonzept.

Während der Bekanntheitsaufbau noch sehr stark durch Inside-out-Kommunikation geprägt wird, so ist doch frühzeitig der Outside-in ausgerichtete Dialog anzustreben. In jedem Fall gilt: Angesichts der Informationsflut, der sich die Stakeholder ausgesetzt sehen, ist das bloße Senden oder gar Streu- 
en von Botschaften und Informationen auch in der Bekanntmachungsphase ineffektiv. Auf der Basis der oben beschriebenen Stakeholder-Analyse ist die Kommunikation von Anfang auf die relevanten Stakeholder auszurichten. Viele der relevanten Stakeholder nutzen Online- und Social-Media-Kommunikationskanäle. Der direkte persönliche Kontakt oder indirekte über Empfehlungen ist selbstredend von besonderem Wert. Dabei ist immer der Grundsatz der „Kommunikation auf Augenhöhe“ zu beachten. Dafür ein Beispiel: Entscheider in Organisationen messen dem Rat von Entscheidern anderer Organisationen hohe Bedeutung zu. Oder sie folgen den Empfehlungen ihres Stabes oder den Berichten der verantwortlichen Mitarbeiter in den Linien-Funktionen (Oltmanns 2008). Bestehen also bei der Forschungseinrichtung keine direkten Kontakte zum Entscheider, ist der Kontakt über die Mitarbeiter aus dessen Organisation oder die Entscheider anderer Organisationen herzustellen, zu denen persönliche Verbindungen bestehen. Solche unterstützenden Verbindungen zu identifizieren, ist ein wesentlicher Nutzen der Stakeholder-Analyse.

Was bei der Entwicklung integrierter operativer Kommunikations-Konzepte für einen Forschungsverbund zu beachten ist, wird an anderer Stelle dieses Buches behandelt.

\subsection{Zusammenfassung}

Um den Herausforderungen eines sich zunehmend ausdifferenzierenden Wissenschafts-Markts in der Gesundheitsforschung durch Globalisierung, Wissensgenerierung, Vernetzung und Informationsflut gerecht $\mathrm{zu}$ werden, bedarf es eines ganzheitlichen Marketing- und pro-aktiven, Dialogorientierten Kommunikationsverständnisses auf Seiten der Führung eines Forschungsverbundes. Stakeholder- und Markenmanagement sind dabei strategische Kommunikations-Aufgaben und stellen die Grundlagen aller operativen Kommunikations-Konzepte und -Maßnahmen dar. Angesichts knapper Ressourcen konzentriert sich die Kommunikation auf relevante Stakeholder. Sie zu identifizieren und mögliche Veränderungen bei der Relevanz für den Forschungsverbund zu berücksichtigen, ist Aufgabe eines Stakeholder-Managements.

Das Markenmanagement verfolgt das Ziel, auf Basis von Positionierung und Leitbild des Forschungsverbundes mit Hilfe der Kommunikation konkrete Vorstellungsbilder in der Wahrnehmung der Stakeholder zu entwickeln, wobei Marken-Identität und -Image so kongruent wie möglich sein sollen. Eine starke Forschungsverbunds-Marke erleichtert den Stakeholdern die Auswahl unter den Wettbewerbern und wird von ihnen als Qualitätsversprechen und -Sicherung gewertet. Zusätzlich zu diesem Crundnutzen trägt sie zu einem „psychologischen Zusatznutzen“ auf Seiten der Stakeholder bei: zur Identifikations-, Image- und Statusbildung, wodurch Loyalität und Bindung mit 


\section{dem Forschungsverbund sowie die Bereitschaft zu dessen Weiterempfehlung gefördert werden.}

\section{Quellenangaben}

Burmann Ch., Halaszovich T., Hemmann F. (2012): Identitätsbasierte Markenführung, SpringerGabler Covey S.R. (1989/2005): Die sieben Wege zur Effektivität, Gabal-Verlag

Freeman R., Reed E., David L. (1983): Stockholders and Stakeholders: A New Perspective on Corporate Governance, in: California Management Review, 25. Ig., H. 3, 1983, S. 88-106

Fritz W. (1996): Marketing als Konzeption des Wissenschaftsmanagements, Wissenschaftsmanagement Ausgaben 1 und 2, 1996

Gad T. (2005): 4D-Branding, MI-Verlag

Janisch M. (1992): Das strategische Anspruchsgruppenmanagement. Vom Shareholder Value zum Stakeholder Value, Diss. St. Gallen

Kotler P., Armstrong G., Wong V., Saunders ). (2011): Grundlagen des Marketings, Pearson, 5. Auflage

Kotler P., Kartajaya H., Setiawan I. (2010): Marketing 3.0 - Die neue Dimension des Marketings, Campus-Verlag Kotler P., Lee N. (2010): Social Marketing für eine bessere Welt, MI-Verlag

Lichtensteiner H., Purtschert R. (2014): Marketing für Verbände und weitere Nonprofit-Organisationen, HauptVerlag, 3. Auflage

Müller-Stewens G., Lechner Ch., Stahl H.K. (2001/2003): Die Gestaltung von Stakeholderbeziehungen als Grundlagen jedes Grenzmanagements in Hinterhuber, Stahl: Fallen die Unternehmensgrenzen? Beiträge zur Außenorientierung der Unternehmensführung, Renningen, S. 270-291

Oltmanns T. (2008): Eliten-Marketing - Wie Sie Entscheider erreichen, Campus-Verlag

Raffée H., Fritz W., Wiedmann P. (1994): Marketing für öffentliche Betriebe, Kohlhammer

Savage G.T., et al. (1991): Strategies for Assessing and Managing Organizational Stakeholders, in: Academy of Management Executive, 5. Ig., H. 2, 1991, S. 61-75

Scheier Chr., Held D. (2007): Was Marken erfolgreich macht, Haufe

\section{Weiterführende Literatur}

Bartsch S., Blümelhuber Ch. (2015): Always Ahead im Marketing. Offensiv, digital, strategisch, Springer Gabler Bruhn M. (2011): Marketing für Nonprofit-Organisationen, Kohlhammer, 2. Auflage

Covey S.R. (2004): Der achte Weg - Mit Effektivität zu wahrer Größe, Gabal-Verlag

Covey S.R. (2012): Die 3. Alternative, Gabal-Verlag

Esch F.R. (2013): Moderne Markenführung, Gabler-Verlag

Kotler P., Keller K.L., Opresnik M.O. (2015): Marketing-Management, Pearson, 14. Auflage

Koziol K., Pförtsch W., Heil S., Albrecht K. (2006): Social Marketing - Erfolgreiche Marketing-Konzepte für Nonprofit-Organisationen

Menz F., Stahl H.K. (2008): Handbuch Stakeholderkommunikation, Erich Schmidt Verlag

Schmidt B.F., Lyczek B. (2005): Unternehmenskommunikation, Gabler-Verlag

Theuvsen L. (2001): Stakeholder-Management - Möglichkeiten des Umgangs mit Anspruchsgruppen, Münsteraner Diskussionspapiere zum Nonprofit-Sektor - Nr. 16

Töpfer A., Braun G.E. (1989): Marketing im staatlichen Bereich, Bonn Aktuell

Tropp J. (2011): Moderne Marketing-Kommunikation, VS Verlag

Wesselmann S., Hohn B. (2012): Public Marketing - Marketing-Management für den öffentlichen Sektor, SpringerGabler 